\title{
Vitamin E as an Adjuvant Treatment for Non- alcoholic Fatty Liver Disease in Adults: A Systematic Review of Randomized Controlled Trials
}

\author{
Muhammad Usman ${ }^{1,2}$, Nabiyah Bakhtawar ${ }^{3}$ \\ 1. Internal Medicine, Leicester Royal Infirmary, Leicester, GBR 2. Internal Medicine, Kettering General Hospital, \\ Kettering, GBR 3. Surgery, Lister Hospital, Stevenage, GBR
}

Corresponding author: Muhammad Usman, usman92yasin@gmail.com

\begin{abstract}
Nonalcoholic fatty liver disease (NAFLD) is one of the most common causes of chronic liver disease. It is characterized by a variety of pathologies, ranging from benign fatty liver to extensive fibrosis and even hepatocellular cancer. Among the several potential risk factors, insulin resistance and increased oxidative stress are the most important. Vitamin $\mathrm{E}$ is an antioxidant with a potential to be used as a treatment for NAFLD. Therefore, we carried out a structured systematic review of all RCTs conducted between 2010 and January 2020. After screening, eight RCTs were included. Our systematic review showed that vitamin E has clinical utility in improving biochemical (ALT and AST levels) and histological abnormalities in NAFLD (hepatic steatosis and lobular inflammation). However, vitamin E does not seem to have significant effects on liver fibrosis. Still, vitamin E has the potential to be used as an adjuvant for the treatment of NAFLD, and its use in clinical practice should be advocated.
\end{abstract}

Categories: Internal Medicine, Gastroenterology

Keywords: tocopherol, nafld, vitamin e, non-alcoholic fatty liver disease, antioxidant, oxidative stress, systematic review

\section{Introduction And Background}

Non-alcoholic fatty liver disease (NAFLD) is an umbrella term used to represent a variety of liver pathologies, ranging from benign non-alcoholic fatty liver (NAFL) to progressive non-alcoholic steatohepatitis (NASH) with or without fibrosis, NASH cirrhosis, and hepatocellular carcinoma (HCC) [1-2]. NAFLD is a diagnosis of exclusion and is characterized by $\geqslant 5 \%$ hepatic fat accumulation in the absence of a history of drinking and other causes of chronic liver disease such viral hepatitis, hemochromatosis, Wilson's disease, autoimmune hepatitis, drug-induced hepatitis, and chronic hepatitis due to endocrine or hereditary causes [2].

Received 06/21/2020

Review began 06/25/2020 Review ended 06/27/2020 Published 07/06/2020

(c) Copyright 2020

Usman et al. This is an open access article distributed under the terms of the Creative Commons Attribution License CC-BY 4.0., which permits unrestricted use, distribution, and reproduction in any medium, provided the original author and source are credited.
The pathogenesis of NAFLD is poorly understood, but it is likely to be a multi-factorial, multi-step, and progressive disease. It is quite likely that metabolic syndrome remains the epicenter of the disease [2]. In addition to insulin resistance, increased production of reactive oxygen species (ROS), oxidative stress, and mitochondrial dysfunction are the key pathologies, leading to biochemical and histological derangements seen in NAFLD [3].

The risk factors established to play a role in the pathogenesis of this disease include central obesity, hyperglycemia, hypertriglyceridemia, and hypertension [1-4]. Other potential risk factors that are being investigated include genetic factors (PNPLA3 and TM6SF2 genes), dysbiosis of gut microbiota, dietary modifications (such as high fructose consumption), and endocrine issues (such as hypothyroidism, hypogonadism, and hypopituitarism) [2-4].

There has been a staggering increase in the number of NAFLD cases over the past 20 years. The prevalence varies according to the region and can be as low as $13.5 \%$ of all liver disease patients in Africa compared to $46 \%$ in America [5-6]. Furthermore, the disease prevalence can be as high as $55.5 \%$ in type II diabetics [7].

Lifestyle modifications are the first-line management for NAFLD. Among the pharmacological agents, pioglitazone and vitamin $\mathrm{E}$ have shown some promise [8]. Vitamin $\mathrm{E}$ is a potent antioxidant that improves biochemical and histological abnormalities associated with NAFLD in various studies [9-10].

Despite the potential benefits of vitamin $\mathrm{E}$ for the treatment of NAFLD and the randomized controlled trials (RCTs) conducted to validate this fact, there is a lack of systematic reviews that could help conclude these studies. There has been some work where authors have looked at the effects of vitamin $\mathrm{E}$ among all population groups [11]. However, there exists a great deal of disproportionation between the prevalence of 
There are a limited number of systematic reviews that specifically look into the effects of vitamin $\mathrm{E}$ on NAFLD outcomes for the adult population. Therefore, this systematic review aims to look at the therapeutic potential of vitamin E used as an adjuvant for NAFLD treatment in the adult population.

\section{Review \\ Methods}

Information Sources

Two independent reviewers (UM, BN) carried out a rigorous literature review using electronic databases. The PRISMA guidelines for carrying out a systematic review were followed. PubMed, Google Scholar, Medline, EMBASE, CENTRAL, and clinical trial directories were searched for peer-reviewed randomized control trials conducted between 2010 and January 2020. Two search themes were used to search the databases that were combined using the Boolean operator 'AND'. For the theme of 'NAFLD', we used keywords such as NAFLD, $\mathrm{NASH}$, liver cirrhosis, and liver disease. For the theme 'vitamin E', we used keywords such as vitamin E, tocotrienol, and tocopherol.

Inclusion Criteria

The search was only limited to RCTs, full-text publications, articles published in English, and studies recruiting adult population (age $\geqslant 18$ ). Trials that investigated the effects of vitamin E on biochemical markers (ALT, AST) and/or histological markers (steatosis, ballooning, inflammation, and fibrosis) were considered

Exclusion Criteria

The exclusion criteria included (1) all studies conducted on children and adolescent population, (2) studies involving individuals with liver disease due to alcohol or secondary causes such as viral hepatitis, hemochromatosis, Wilson's disease, autoimmune hepatitis, drug-induced hepatitis, and chronic hepatitis due to endocrine or hereditary causes, and (3) studies that were not RCTs were excluded from the study.

Data Extraction and Study Selection

Both the researchers carried out an individual review of the literature. All results were compiled and both researchers compared their data and solved any conflicts through mutual concurrence and consultation.

A total of 345 studies were identified through a literature search. A total of 290 studies were excluded as they were in different languages, were not full-text studies, and because of their study design (included adolescents/children). The researchers read through 55 studies manually and excluded another 47 after reading through the article titles and abstracts. A total of 8 RCTs were included in the final analysis.

Figure 1 describes the literature review process. 


\section{Cureus}

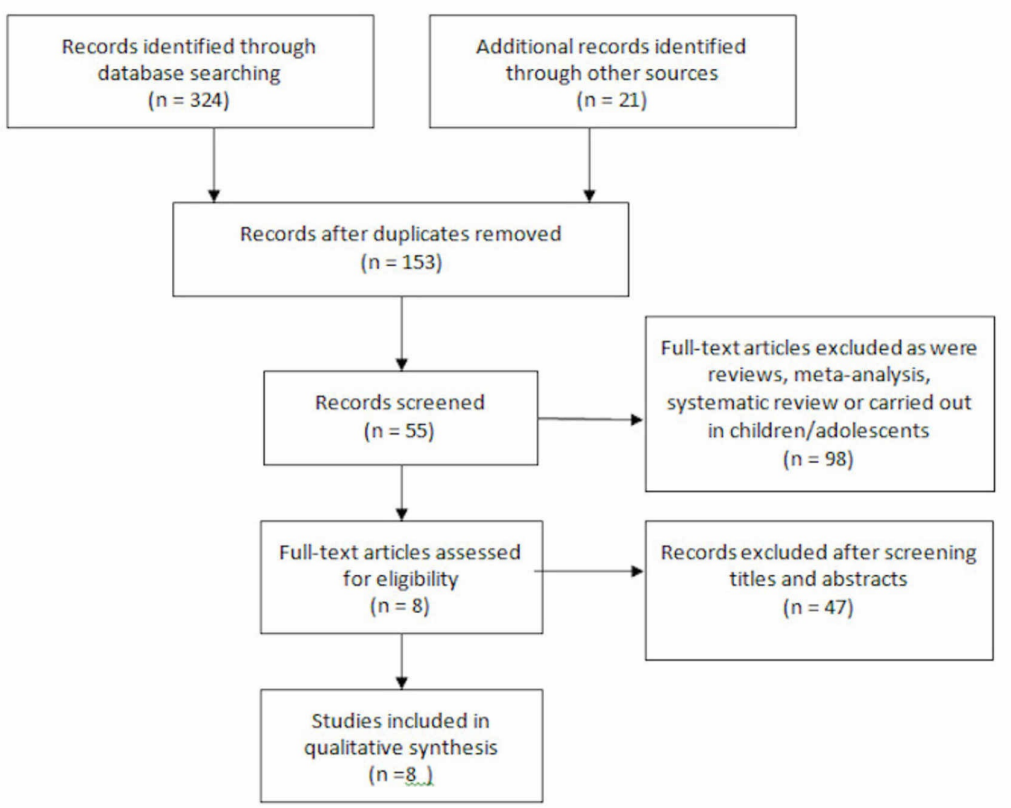

FIGURE 1: Flowchart based on PRISMA guidelines describing study identification and selection process

\section{Results}

A total of eight RCTs published between 2010 and January 2020 were identified. All the trials included a total of 1,227 participants with the mean age ranging between 44.3 and 59 years. The duration of studies ranged from 3 months to 52 months. Table 1 includes the RCTs added in chronological order and summarizes the population demographics and interventions used. 


\section{Cureus}

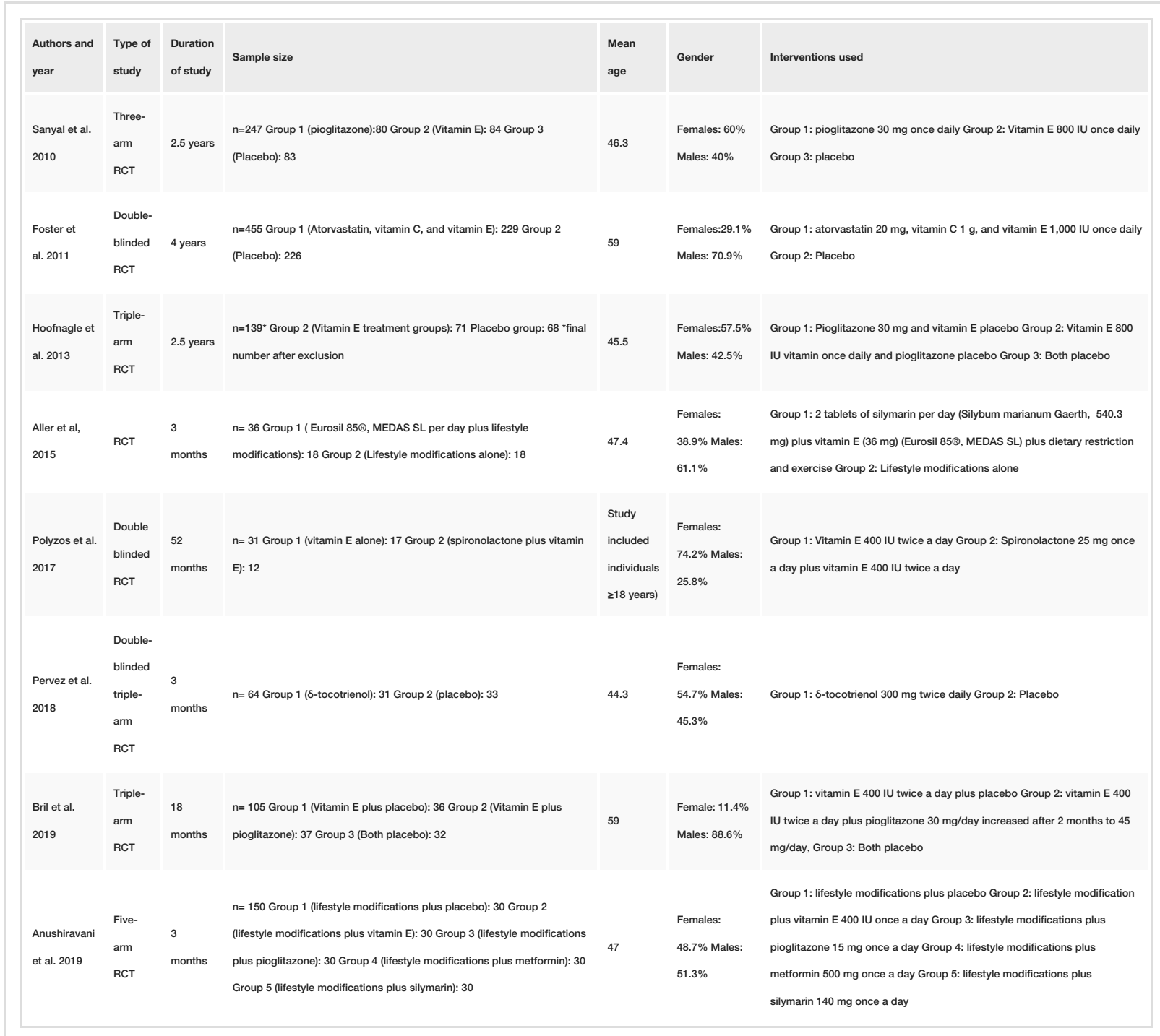

TABLE 1: Summary of patient demographics, study design, and study interventions in the included RCTs

$\mathrm{RCT}$, randomized controlled trials

The study by Aller et al. did not clearly define the randomization strategy and included a combination therapy of vitamin E and silymarin tested against lifestyle modifications [16]. One double-blinded RCT compared a combination therapy of atorvastatin, vitamin C, and vitamin E and placebo [14]; the other double-blinded study compared vitamin E monotherapy and vitamin E combination therapy with spironolactone [17], and another compared $\delta$-tocotrienol monotherapy therapy and placebo [18]. Three studies were triple-arm double-blinded RCTs where at least one group was given vitamin $\mathrm{E}$ as a monotherapy $[13,15,19]$. Anushiravani et al. carried out a five-arm double-blinded RCT, where vitamin E monotherapy was used in one group [20].

All the RCTs used varying doses of vitamin E, ranging from 400 IU to 1,000 IU [13-15,17,19-20]. Aller et al. used $36 \mathrm{mg}$ vitamin E daily [16], and Pervez et al. used $600 \mathrm{mg}$ ठ-tocotrienol (an isoform of vitamin E) daily [18].

Pioglitazone was the most common co-intervention used in four of the eight RCTs [13,15,19-20]. This was followed by silymarin in two [16,20], lifestyle modifications in two [16,20], vitamin C in one [14], atorvastatin in one [14], spironolactone in one [17], and metformin in one of the studies [20].

Table 2 summarizes the effects of vitamin E on biochemical and histological markers of NAFLD. 


\section{Cureus}

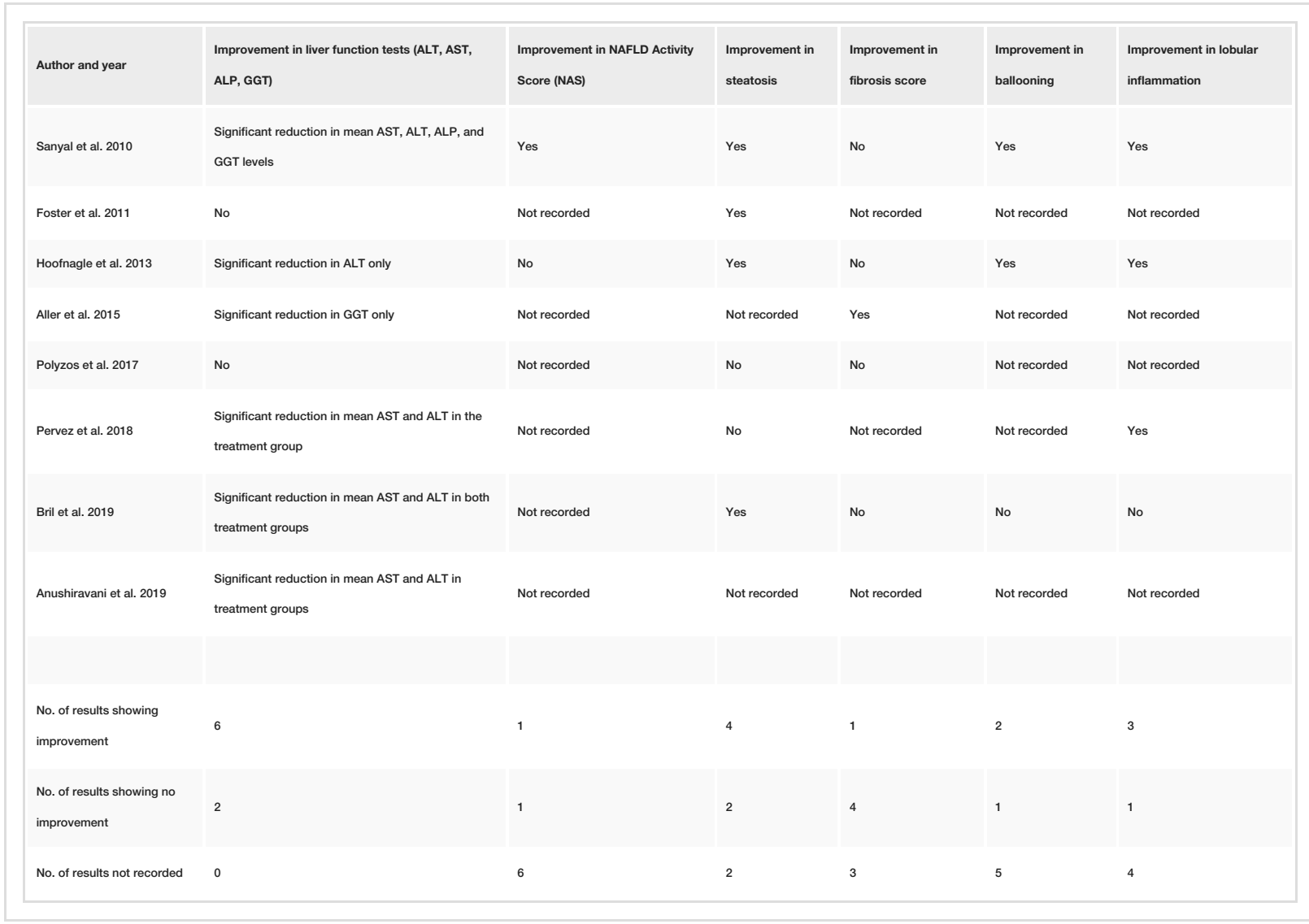

\section{TABLE 2: Table summarizing the effects vitamin E on biochemical and histological markers of NAFLD}

NAFLD, nonalcoholic fatty liver disease; ALT, alanine aminotransferase; AST, aspartate aminotransferase; ALP, alkaline phosphatase; GGT, gammaglutamyl transferase

Six of the eight RCTs noted significant improvements in liver function tests [13,15-16,18-20]. Four studies noted a significant improvement in both ALT and AST [13,18-20]; one noted improvement in ALT only [15], and one reported improvement in GGT only [16]. Sanyal et al. reported additional improvements in ALP and GGT levels [13]. Foster et al. and Polyzos et al. did not report any improvements in liver function tests with vitamin E therapy [14-17].

When liver histological parameters were evaluated, vitamin E treatment groups showed only limited improvements. Four RCTs showed improvement in steatosis [13-15,19], and three showed improvement in inflammation $[13,15,18]$. Sanyal et al. and Hoofnagle et al. reported an improvement in hepatocyte ballooning [13,15]. Only Aller et al. showed improvement in fibrosis [16], and Sanyal et al. showed improvement in NAFLD Activity Score (NAS) [13].

However, it must be noted that several markers of hepatic histological response (such as NAS, hepatocyte ballooning, and hepatocyte inflammation) were not evaluated in several studies [14-20] (Table 2).

\section{Discussion}

Insulin resistance, metabolic syndrome, and oxidative stress are the key pathologies in NAFLD. Lifestyle modifications consisting of diet, exercise, and weight loss are still considered the first-line treatments for NAFLD [21-22]. However, the attrition and dropout rates during lifestyle modification regimens can be as high as $40 \%$ and 50\%, respectively [23]. According to the European Association for the Study of the Liver (EASL), pharmacological therapies should be considered in cases of progressive NAFLD where lifestyle modifications are producing inadequate results [24]. Vitamin E is being currently recommended for the treatment of NAFLD by the American Association for the Study of Liver Diseases (AASLD) and the National Institute for Health and Care Excellence (NICE), UK [25]. 
Mitochondrial dysfunction and endoplasmic reticulum stress lead to increased lipid peroxidation. This causes an imbalance in pro-oxidants and antioxidants leading to increased oxidative stress and liver dysfunction in NAFLD [3]. In the context of NAFLD, vitamin E exerts a variety of effects. It acts as an antioxidant and reduces reactive oxygen species; increases the antioxidants like glutathione and superoxide dismutase, and lowers the hepatic inflammation and fibrosis by reducing the expression of pro-apoptotic (Bax), TGF- $\beta$, COX-2, and MMP-2 genes [9].

Erhardt et al. 2011 noted that serum levels of vitamin E in NAFLD patients were lower than controls [26]. Our meta-analysis showed the potential of vitamin $\mathrm{E}$ in the treatment of NAFLD. Our study showed that vitamin E leads to significant improvement in liver function test derangements seen in NAFLD, especially in AST and ALT levels. Our study is consistent with the current literature were similar findings were noted in previous meta-analyses $[2,9]$.

However, the effects of vitamin E on liver histological parameters in NAFLD patients have been reported inconsistently in the literature. Our analysis showed that vitamin E is only able to improve hepatic steatosis and hepatic inflammation. It does not significantly improve liver fibrosis or other histological markers of disease activity like hepatocyte ballooning. Different findings have been reported in the literature, where Xu et al. 2015 reported a significant improvement in all histological indicators of liver injury including liver fibrosis, lobular inflammation, ballooning, and steatosis with the use of vitamin E. However, that metaanalysis included only three RCTs [27]. However, our findings have been validated by subsequently done more comprehensive meta-analyses and systematic reviews showing that vitamin E has limited efficacy in improving hepatic fibrosis. It might have some benefit in improving steatosis and lobular inflammation $[2,9]$.

Limitations

It must be noted that the RCTs included in our systematic review have some limitations. There was a lack of standardization in the dose, form, and frequency of vitamin E used in the RCTs. The sample sizes in the current RCTs were relatively small. Moreover, the RCTs did not look into one or more significant factors useful for monitoring disease progression in NAFLD.

\section{Conclusions}

Vitamin E is a potent antioxidant useful for the management of NAFLD. Our systematic review suggests that vitamin E helps improve several biochemical and histological derangements in NAFLD. However, it does not seem to improve some important aspects of NAFLD disease pathology such as hepatic fibrosis. Still, vitamin E has a significant potential to improve biochemical and histological indicators of NAFLD, especially when used as an adjuvant to other therapies such as lifestyle modification and pharmacotherapies such as pioglitazone. Therefore, vitamin E should be more commonly used in clinical practice as an adjuvant to the mainstay treatments for the management of NAFLD.

\section{Additional Information \\ Disclosures}

Conflicts of interest: In compliance with the ICMJE uniform disclosure form, all authors declare the following: Payment/services info: All authors have declared that no financial support was received from any organization for the submitted work. Financial relationships: All authors have declared that they have no financial relationships at present or within the previous three years with any organizations that might have an interest in the submitted work. Other relationships: All authors have declared that there are no other relationships or activities that could appear to have influenced the submitted work.

\section{References}

1. Cobbina E, Akhlaghi F: Non-alcoholic fatty liver disease (NAFLD) - pathogenesis, classification, and effect on drug metabolizing enzymes and transporters. Drug Metab Rev. 2017, 49:197-211. 10.1080/03602532.2017.1293683

2. Perumpail BJ, Khan MA, Yoo ER, et al.: Clinical epidemiology and disease burden of nonalcoholic fatty liver disease. World J Gastroenterol. 2017, 23:8263-8276. 10.3748/wig.v23.i47.8263

3. Masarone M, Rosato V, Dallio M, et al.: Role of oxidative stress in pathophysiology of nonalcoholic fatty liver disease. Oxid Med Cell Longev. 2018, 2018:9547613. 10.1155/2018/9547613

4. Arab PJ, Arrese M, Trauner M: Recent insights into the pathogenesis of nonalcoholic fatty liver disease . Annu Rev Pathol. 2018, 13:321-350. 10.1146/annurev-pathol-020117-043617

5. Younossi ZM, Koenig AB, Abdelatif D, et al.: Global epidemiology of nonalcoholic fatty liver disease-Metaanalytic assessment of prevalence, incidence, and outcomes. Hepatology. 2016, 64:73-84. 10.1002/hep.28431

6. Vernon G, Baranova A, Younossi ZM: Systematic review: the epidemiology and natural history of nonalcoholic fatty liver disease and non-alcoholic steatohepatitis in adults. Ailment Pharmacol Ther. 2011, 34:274-85. 10.1111/j.1365-2036.2011.04724.x

7. Younossi ZM, Golabi P, Avila LD, et al.: The global epidemiology of NAFLD and NASH in patients with type 2 diabetes: a systematic review and meta-analysis. J Hepatol. 2019, 71:793-801. 10.1016/j.jhep.2019.06.021 
8. Hung KC, Bodenheimer Jr HC: Current treatment of nonalcoholic fatty liver disease/nonalcoholic steatohepatitis. Clin Liver Dis. 2018, 22:175-187. 10.1016/j.cld.2017.08.012

9. Hadi HE, Vettor E, Rossato M: Vitamin E as a treatment for nonalcoholic fatty liver disease: reality or myth? . Antioxidants (Basel). 2018, 7:12. 10.3390/antiox7010012

10. Pacana T, Sanyal AJ: Vitamin E and non-alcoholic fatty liver disease . Curr Opin Clin Nutr Metab Care. 2012, 15:641-648. 10.1097/MCO.0b013e328357f747

11. Amanullah I, Khan YH, Anwar I, et al.: Effect of vitamin E in non-alcoholic fatty liver disease: a systematic review and meta-analysis of randomised controlled trials. Postgrad Med J. 2019, 95:601-611. 10.1136/postgradmedj-2018-136364

12. Nobili V, Alisi A, Newton KP, et al.: Comparison of the Phenotype and Approach to Pediatric vs Adult Patients With Nonalcoholic Fatty Liver Disease. Gastroenterology. 2016, 150:1798-1810. 10.1053/j.gastro.2016.03.009

13. Sanyal AJ, Chalasani N,Kowdley KV, et al.: Pioglitazone, Vitamin E, or Placebo for Nonalcoholic Steatohepatitis. N Engl J Med. 2010 May 6, 362:1675-1685. 10.1056/NEJMoa0907929

14. Foster T, Budoff MJ, Saab S, et al.: Atorvastatin and antioxidants for the treatment of nonalcoholic fatty liver disease: The St Francis heart study randomized clinical trial. Am J Gastroenterol. 2011, 106:71-77. 10.1038/ajg.2010.299

15. Hoofnagle JH, Natta ML, Kleiner DE, et al.: Vitamin E and changes in serum alanine aminotransferase levels in patients with non-alcoholic steatohepatitis. Aliment Pharmacol Ther. 2013 Jul, 38:10.1111/apt.12352

16. Aller R, Izaola O, Gomez S, et al.: Effect of silymarin plus vitamin E in patients with non-alcoholic fatty liver disease. A randomized clinical pilot study. Eur Rev Med Pharmacol Sci. 2015, 19:3118-3124.

17. Polyzos SA, Kountouras J, Mantzoros CS, et al.: Effects of combined low-dose spironolactone plus vitamin E vs vitamin E monotherapy on insulin resistance, non-invasive indices of steatosis and fibrosis, and adipokine levels in non-alcoholic fatty liver disease: a randomized controlled trial. Diabetes Obes Metab. 2017, 19:1805-1809. 10.1111/dom.12989

18. Pervez MA, Khan DA, Ijaz A, et al.: Effects of delta-tocotrienol supplementation on liver enzymes, inflammation, oxidative stress and hepatic steatosis in patients with nonalcoholic fatty liver disease. Turk J Gastroenterol. 2018 Mar, 29:170-176. 10.5152/tjg.2018.17297

19. Bril F, Biernacki DM, Kalavalapalli S, et al.: Role of vitamin E for nonalcoholic steatohepatitis in patients with type 2 diabetes: a randomized controlled trial. Diabetes Care. 2019, 42:1481-1488. 10.2337/dc19-0167

20. Anushiravani A, Haddadi N, Pourfarmanbar M, et al.: Treatment options for nonalcoholic fatty liver disease . Eur J Gastroen Hepat. 2019, 31:613-617. 10.1097/meg.0000000000001369

21. Haufe S, Engeli S, Kast P, et al.: Randomized comparison of reduced fat and reduced carbohydrate hypocaloric diets on intrahepatic fat in overweight and obese human subjects. Hepatology. 2011, 53:15041514. 10.1002/hep.24242

22. Asrih M, Jornayvaz FR: Diets and nonalcoholic fatty liver disease: the good and the bad . Clin Nutr. 2014, 33:186-190. 10.1016/j.clnu.2013.11.003

23. Lin Y, Huang H: Eight-week of low-intensive lifestyle modification does improve insulin resistance in adults with metabolic syndrome. Diabetes Metab Syndr Obes. 2019, 12:613-621. 10.2147/DMSO.S201526

24. European Association for the Study of the Liver (EASL: European Association for the Study of Diabetes (EASD); European Association for the Study of Obesity (EASO). EASL-EASD-EASO Clinical Practice Guidelines for the management of non-alcoholic fatty liver disease. J Hepatol. 2016, 64:1388-1402. 10.1016/j.jhep.2015.11.004

25. Leoni S, Tovoli F, Napoli L, et al.: Current guidelines for the management of non-alcoholic fatty liver disease: a systematic review with comparative analysis. World J Gastroenterol. 2018, 24:3361-3373. 10.3748/wjg.v24.i30.3361

26. Erhardt A, Stahl W, Sies H, et al.: Plasma levels of vitamin E and carotenoids are decreased in patients with nonalcoholic steatohepatitis (NASH). Eur J Med Res. 2011, 16:76. 10.1186/2047-783X-16-2-76

27. Xu R, Tao A, Zhang S, et al.: Association between vitamin E and non-alcoholic steatohepatitis: a metaanalysis. Int J Clin Exp Med. 2015, 8:3924. 